\section{Antiinflammatory eggs}

Proteins secreted by parasite eggs might hold the key to the next antiinflammatory therapy. On page 1319, Smith and colleagues describe the first chemokine binding protein (CKBP) produced by a human pathogen and show that it can suppress acute inflammation in disease models.

The blood-dwelling worm Schistosoma mansoni takes advantage of the host's immune system in two ways. It subverts specific inflammatory cells to help move its eggs from the intestinal vasculature to the feces, where they can be shed. It also suppresses the immune response in order to establish chronic infections, in part as shown here by keeping other inflammatory cells away from the eggs.

This selective cell recruitment was not due solely to cytokine modulation, a known schistosome ability. As viruses produce chemokine binding proteins to neutralize host chemokines, the authors investigated whether S. mansoni might also make a chemokine binding protein to mask the eggs from strong inflammation.

Smith and colleagues now identify a novel CKBP (smCKBP) that is secreted from schistosome eggs. This protein bound to the chemokines CXCL8 and CCL3 and prevented them from binding to their receptors on immune cells. Injecting mice with recombinant smCKBP blocked chemokinemediated neutrophil infiltration in response to a variety of inflammatory stimuli. This treatment, however, had no effect on chronic inflammation disorders such as arthritis.

The authors suggest that, in nature, the parasite eggs secrete smCKBP to keep neutrophils and macrophages out of the surrounding granuloma so as not to attract immune attention, but pinning this mechanism down will require further study. Already tolerated by infected humans, smCKBP holds great potential as a new therapy for acute inflammatory diseases, especially those caused by neutrophilia. JEM

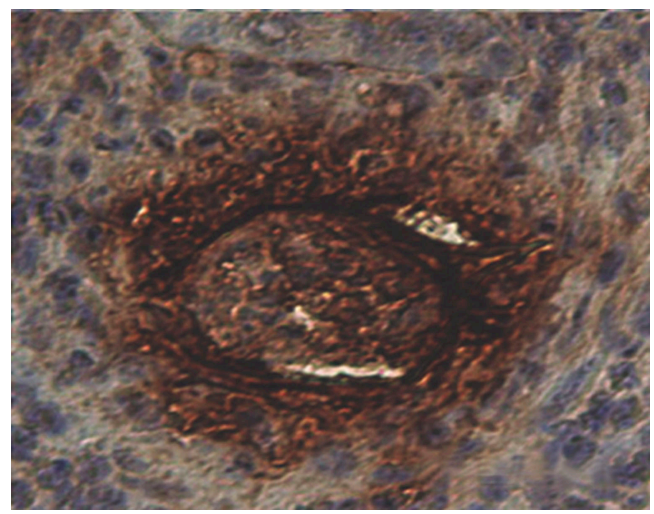

A schistosome egg secretes chemokine binding protein (brown) to ward off immune cells.

\section{Toll-free autoimmunity}

Self-DNA and pathogen-derived DNA take different routes to immune activation, say Okabe and colleagues (page 1333). Thus, it may be possible to block an autoimmune response to self-DNA without compromising the beneficial response to pathogenic DNA.

Microbial DNA is mopped up by macrophages and dendritic cells, which get activated via Toll-like receptors (TLRs), resulting in the production of interferon- $\beta$ (IFN $\beta$ ). Recent studies have shown that mammalian DNA complexed to antibodies can also trigger IFN $\alpha$ production via a TLR (specifically TLR9).

The immune system can also be activated by naked self-DNA, leading to some autoimmune diseases. One such state occurs in mice lacking DNaseII. These mice accumulate undigested self-DNA from dead cells in their macrophages, and act as a model for systemic lupus erythematosus (SLE), a disease characterized by autoantibodies to circulating self-DNA.

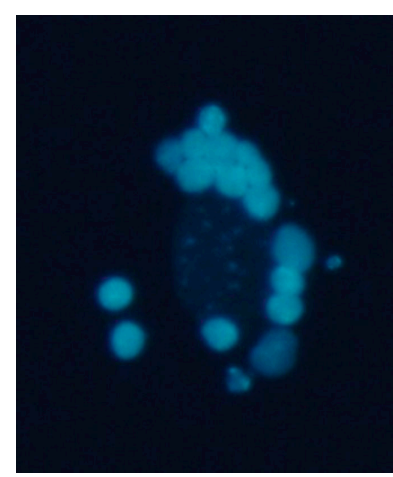

Self-DNA (blue) activates DNasell-deficient macrophages via a unique pathway.
The up-regulation of IFN $\beta$ in these mice kills precursor red blood cells, induces severe anemia, and causes the observed embryonic lethality. A DNaseII-IFN receptor double knock-out rescued these effects. In the current study, however, the DNaseII ${ }^{-}$phenotype, including the IFN $\beta$ up-regulation, is not rescued by knockout of TLR9, TLR3, or their adaptor molecules MyD88 and TRIF.

The results imply that a TLR-independent pathway of IFN activation exists. Therefore, it may be possible to block that autoimmune response pathway while leaving the TLRdependent response to pathogens intact. JEM

\section{A plague of deubiquitination}

Plague bacteria inject infected host cells with a ubiquitin-chopping enzyme, according to Zhou and colleagues in a study on page 1327. The group shows that the virulence factor YopJ operates as a deubiquitinase to shut down multiple signaling pathways that would otherwise help trigger an inflammatory immune response.

Pathogenic Yersinia bacteria need the cysteine protease YopJ to kill infected macrophages and promote systemic infection in mice. YopJ's activity somehow inhibits pro-inflammatory NF-кB signaling. Previous work by others had shown that YopJ can catalyze the removal of the ubiquitin-like protein SUM0-1 from cellular proteins. That work proposed that YopJ might desumolyate upstream activators of NF- $\mathrm{KB}$.

Here, the authors show that YopJ does not act as a SUMO-1 isopeptidase, but rather as a deubiquitinase. YopJ stripped the ubiquitin chains from the adaptor molecules TRAF2 and TRAF6, which need these modifications to help activate $\mathrm{NF}-\kappa \mathrm{B}$ signaling, and also from the NF- $\mathrm{KB}$ inhibitor protein $1 \kappa \mathrm{B}$. Ubiquitination of I $\mathrm{B}$ normally tags the inhibitor for proteasomal degradation, thus allowing NF- $\kappa B$ to move into the nucleus and activate inflammatory genes.

YopJ could clip ubiquitin molecues attached either by their lysine 63 residue (as

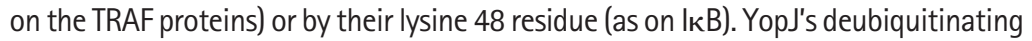
"promiscuity," the authors write, allows the bacteria to simultaneously shut off multiple routes of NF- $\mathrm{B}$ activation and thus to avoid immune attack. YopJ-related proteins exist in a diverse variety of bacterial pathogens, indicating that encoding deubiquitinases may be a common immune evasion tactic. JEM 